Universitäre Psychiatrische Dienste, Direktion Alterspsychiatrie, Bern'; Institut für Rechtsmedizin der Universität Zürich, Verkehrsmedizin und Forensische Psychiatrie $^{2}$; Staatsanwaltschaft Zürich-Limmat ${ }^{3}$; Stadtspital Waid, Klinik für Akutgeriatrie, Memory-Klinik, Zürich ${ }^{4}$; Neurologie Spitalzentrum Biel'; Universitätsspital Basel, Akutgeriatrie, Memory Clinic, Basel ${ }^{6}$; Schweizerische Alzheimervereinigung, Yverdon-les-Bains ${ }^{7}$; Inselspital, Abteilung für Kognitive und Restorative Neurologie, Bern ${ }^{8}$; Universität Bern, Gerontechnologie und Rehabilitation, Bern ${ }^{9}$; Strassenverkehrsamt Chur ${ }^{10}$; Service Universitaire de Psychiatrie de l'Age Avancé, Départment de Psychiatrie, Centre Hospitalier Universitaire Vaudois, Lausanne"; Praxisgemeinschaft Kofmehl-Huus, Subingen ${ }^{12}$

1,9U.P. Mosimann, ${ }^{\mathrm{J} J}$. Bächli-Biétry, ${ }^{3}$ J. Boll, ${ }^{4} \mathrm{I}$. Bopp-Kistler, ${ }^{5} \mathrm{~F}$. Donati, ${ }^{6}$ R.W. Kressig, ${ }^{7}$ B. Martensson, ${ }^{6}$ A.U. Monsch, ${ }^{8,9}$ R. Müri, ${ }^{9}$ T. Nef, ${ }^{10}$ A. Rothenberger, ${ }^{2}$ R. Seeger, ${ }^{11} A$. von Gunten, ${ }^{12} \mathrm{U}$. Wirz

\title{
Konsensusempfehlungen zur Beurteilung der medizinischen Mindestanforderungen für Fahreignung bei kognitiver Beeinträchtigung
}

\author{
Consensus Recommendations for the Assessment of Fitness to Drive in \\ Cognitively Impaired Patients
}

\section{Zusammenfassung}

Die Memory Clinics sind auf evidenzbasierte Demenzdiagnostik und Therapie spezialisiert. Bei der Diagnose Demenz sind sie verpflichtet, erkrankte Motorfahrzeuglenker über mögliche Beeinträchtigungen im Strassenverkehr aufzuklären. Patienten und Angehörige brauchen bei dieser schwierigen Fragestellung eine kompetente Beratung, die das Mobilitätsbedürfnis und die Risiken sorgfältig gegeneinander abwägt. Der Zeitpunkt der Demenzdiagnose ist nicht zwingend identisch mit dem Zeitpunkt, an dem die Fahreignung aus medizinischen Gründen nicht mehr gegeben ist. Für die Entscheidung, ob die medizinischen Mindestanforderungen erfüllt sind, müssen die Ursache und der Schweregrad der Demenz, die Komorbiditäten und die Medikation berücksichtigt werden. Im Auftrag des Vereins Swiss Memory Clinics hat eine Expertengruppe praktische Empfehlungen für die Einschätzung der Fahreignung in Memory Clinics erarbeitet.

Schlüsselwörter: Memory Clinic Demenz-Autofahren-Verkehrssicherheit

\begin{abstract}
Allgemeines
Im Verein Swiss Memory Clinics haben sich mehr als 30 Schweizer Memory Clinics zusammengeschlossen (http:// www.swissmemoryclinics.ch/). Der Verein hat u.a. das Ziel, Qualitätsstandards für die Demenzdiagnostik und Therapie zu entwickeln. Memory Clinics bieten spezialisierte Demenzdiagnostik und Therapie an. Der diagnostische Prozess ist interdisziplinär und berücksichtigt natio-
\end{abstract}

\footnotetext{
* Mitglieder der interdisziplinären Expertengruppe Fahreignung bei Demenz des Vereins Swiss Memory Clinics (in alphabetischer Reihenfolge):

Dr. phil. J. Bächli (Verkehrspsychologie); Dr. iur. J. Boll (Staatsanwaltschaft); Dr. med. I. Bopp-Kistler (Geriatrie); Dr. med. F. Donati (Neurologie); Prof. Dr. med. R. W. Kressig (Geriatrie); lic. oec. Birgitta Martensson (Alzheimervereinigung); Prof. Dr. phil. A. U. Monsch (Neuropsychologie); Prof. Dr. med. U. P. Mosimann (Alterspsychiatrie; Vorsitz); Prof. Dr. med. R. Müri (Neurologie); Prof. Dr. sc. T. Nef (Ingenieurwissenschaften); A. Rothenberger (Verkehrsexperte); Dr. med. R. Seeger (Rechtsmedizin); Prof. Dr. med. A. von Gunten (Alterspsychiatrie) und Dr. med. et phil. nat. U. Wirz (Hausarztmedizin).
} 
nale Konsensusempfehlungen [1] und internationale Richtlinien [2]. Memory Clinics arbeiten im Auftrag von niedergelassenen Ärzten und suchen die $\mathrm{Zu}$ sammenarbeit mit Angehörigen. Sie fördern die Vernetzung von Betroffenen und Angehörigen (z.B. Schweizerische Alzheimervereinigung; http://www.alz.ch).

Demenzen sind progrediente Erkrankungen, die abhängig von Ursache und Schweregrad die Fahreignung beeinträchtigen und das Unfallrisiko erhöhen können [3]. Die Inzidenz und Prävalenz von Demenzen nehmen altersabhängig zu. In der Schweiz leben gemäss Hochrechnungen mehr als 110000 Menschen mit Demenz und die meisten von ihnen, d.h. etwa $60 \%$, leben zu Hause [4]. Demenzen werden einerseits unterdiagnostiziert, was dazu führen kann, dass Fahrzeuglenker in Unkenntnis der Erkrankung Motorfahrzeuge lenken. Anderseits stimmt der Zeitpunkt der Diagnose nicht zwingend mit dem Zeitpunkt überein, an dem die Fahreignung aus medizinischen Gründen nicht mehr gegeben ist. Im internationalen Vergleich

Im Artikel verwendete Abkürzungen:

AAN American Academy of Neurology

BGE Bundesgericht

CDR Clinical Dementia Rating

CERAD Consortium to Establish a Registry for Alzheimer's Disease

DLB Dementia with Lewy Bodies

FTD Fronto-temporale Lobärdegeneration

IADL Instrumental Activities of Daily Living

ICADTSInternational Council on Alcohol, Drugs and Traffic Safety

$\mathrm{MCl}$ Mild Cognitive Impairment

MMSE Mini-Mental-Status Examination

MWT Maintenance of Wakefullness Test

NPI Neuropsychiatrisches Inventar

OR Obligationenrecht

PDD Parkinson-Demenz

SVG Schweizerisches Strassenverkehrsgesetz

TAP Test zur Aufmerksamkeitsprüfung

UFOV Useful Field of View Test

VZV Verkehrszulassungsverordnung ist die Verkehrssicherheit in der Schweiz gross [5]. Es fehlen jedoch Statistiken, die aufzeigen, wie viele Senioren mit Demenz Verkehrsunfälle verursachen.

Autofahren ist wohl eine der komplexesten instrumentellen Alltagsaktivitäten (instrumental activities of daily living $[I A D L])$, die ein optimales Zusammenspiel von Perzeption, Kognition und Motorik erfordert. Die häufigsten verkehrsrelevanten Erkrankungen bei Senioren sind Demenzen und altersassoziierte Sehstörungen. Die typischen Fahrschwierigkeiten bei Demenz sind: das Nichteinhalten der Fahrspur, abrupte Spurwechsel, fehlende Geschwindigkeitsgestaltung, unsicheres oder fehlerhaftes Verhalten an Verzweigungen, Nichtgewähren des Vortritts, Nichtbeachten des Rechtsvortritts oder Fehlinterpretation von Lichtsignalen und anderes mehr [6].

Die Kernkompetenz der Memory Clinic liegt darin, eine Demenz zu diagnostizieren, deren Ursache festzustellen und den Schweregrad der Erkrankung zu quantifizieren. Für die Einschätzung, ob trotz Erkrankung die medizinischen Mindestanforderungen erfüllt bleiben, müssen zusätzlich verkehrsrelevante Komorbiditäten und Medikationen mitberücksichtigt werden. Die meisten Informationen für die Beurteilung werden im Rahmen der Demenzdiagnostik erhoben. Der Verzicht auf das Autofahren sollte nicht leichtfertig empfohlen werden, da bei Senioren durch die Einschränkung der eigenen körperlichen Leistungsfähigkeit die Abhängigkeit zum Auto steigt, das Umsteigen auf öffentliche Verkehrsmittel schwierig ist und Angewöhnungszeit braucht. Die Einschränkung der Mobilität kann zudem zu sozialer Isolation, zu Depression oder Institutionalisierung führen $[7,8]$.

Der Verein der Swiss Memory Clinics hat eine interdisziplinäre Expertengruppe beauftragt, praktische Empfehlungen für die Beurteilung der medizinischen Mindestanforderungen bei Demenzen zu erarbeiten. In der Expertengruppe waren die Fachgebiete Geriatrie, Psychiatrie, Neurologie, Verkehrsmedizin, Neuro- psychologie, Verkehrspsychologie, Hausarztmedizin, Strassenzulassungsbehörde, Verkehrstechnik, Jurisprudenz und die Alzheimervereinigung vertreten. Die hier im Konsensus erarbeiteten Empfehlungen fokussieren auf ältere Motorfahrzeuglenker* der Gruppe 3 mit der Führerausweiskategorie B (d.h. Fahrzeugnutzung im Privatverkehr, Fahrzeuge bis 3,5 Tonnen und bis zu acht Sitzplätzen). Bei der Erarbeitung der Empfehlungen hat die Expertengruppe die eigene klinische Erfahrung, die rechtlichen Rahmenbedingungen, die publizierte Evidenz und bestehenden Konsensusempfehlungen [9-11] berücksichtigt.

\section{Die rechtlichen Rahmen- bedingungen}

Die rechtlichen Rahmenbedingungen sind im Schweizerischen Strassenverkehrsgesetz (SVG) (SR 741.01), in der Verordnung über die Zulassung von Personen und Fahrzeugen zum Strassenverkehr (Verkehrszulassungsverordnung (VZV) SR 741.51) und im Obligationenrecht (OR) (SR 220) geregelt (Tab. 1).

Das SVG (Art. 14, Abs. 4) (http://www. admin.ch/ch/d/sr/c741_01.html) regelt das ärztliche Melderecht an die Aufsichtsbehörde oder an die Verkehrszulassungsbehörde. Das Melderecht gilt für Personen, die wegen körperlichen oder geistigen Krankheiten oder wegen Süchten zum sicheren Führen von Motorfahrzeugen nicht mehr fähig sind. Die Gesetzgebung regelt, dass die Meldung im Ermessen des Arztes liegt und dazu keine Entbindung des Berufsgeheimnisses notwendig ist. Es handelt sich hier ausdrücklich um ein Melderecht und keine Meldepflicht. Das Behandlungsteam einer Memory Clinic ist im Rahmen der beruflichen Sorgfaltspflicht (OR Art. 398)

\footnotetext{
Der Lesbarkeit halber wird im ganzen Manuskript die männliche Form, stellvertretend für beide Geschlechter verwendet.
} 
(http://www.admin.ch/ch/d/sr/c220.html) zusätzlich verpflichtet, die Untersuchungen sorgfältig durchzuführen, die Patienten über die Erkrankung, deren Prognose und über die möglichen Auswirkungen im Strassenverkehr aufzuklären. Der Inhalt der Untersuchungsergebnisse und der Aufklärung müssen in der Krankengeschichte dokumentiert werden. Bei schwer amnestischen oder anosognostischen Patienten empfiehlt es sich, den Ausgang der Untersuchung in Anwesenheit der Angehörigen zu besprechen und allenfalls die Empfehlungen schriftlich mitzuteilen.

DieVZV (http://www.admin.ch/ch/d/sr/ c741_51.html) regelt unter anderem die regelmässigen medizinischen Kontrolluntersuchungen (Art. 27), die Kontrollfahrt (Art. 29) und die medizinischen Mindestanforderungen (Anhang 1). Art. 27 sieht bei den über 70-jährigen Ausweisinhabern zurzeit alle zwei Jahre eine medizinische Kontrolluntersuchung vor, die von den meisten Kantonen an die
Hausärztinnen und Hausärzte delegiert wird. Pro Jahr fallen in der Schweiz so ungefähr 175000 Kontrolluntersuchungen an [3]. Falls nach einer Kontrolluntersuchung von der Behörde zusätzliche spezialärztliche Abklärungen empfohlen werden, kann die Behörde eine Zusatzuntersuchung bei Spezialisten, z.B. in einer Memory Clinic oder in einer verkehrsmedizinischen Abklärungsstelle anordnen. In dieser Situation hat die Memory Clinic gegenüber der Behörde Garantenpflicht. Eine vollständige Rückmeldung und Empfehlung an die Behörde ist Teil des Auftrages, der in der Regel vom Exploranden bezahlt werden muss. Da die Memory Clinic in diesem Falle eine Gutachterrolle einnimmt, ist es wichtig und notwendig, dem Exploranden das Auftragsverhältnis, die Kostenfolgen und Verpflichtung der Informationsweitergabe zu erklären.

Die medizinischen Mindestanforderungen (VZV Anhang 1) sehen u.a. vor, dass keine schweren Nervenkrankheiten, keine Geisteskrankheiten von Bedeutung und keine periodische Bewusstseinstrübung oder Bewusstseinsverluste vorliegen dürfen. Der Gesetzgeber erlaubt erhöhtes Risiko. Er bejaht z.B. Autofahren mit 0,5 Promille Alkohol, obwohl dies das Unfallrisiko erhöht. Ähnliches gilt bei Visus- und Gesichtsfeldeinschränkungen, bei denen innerhalb eines gewissen Rahmens die Mindestanforderungen erfüllt bleiben, obwohl das Unfallrisiko ansteigt. Für kognitive Einschränkungen oder Demenzen gibt es einen gewissen Ermessensspielraum, da klar definierte Schwellenwerte fehlen. Die Rechtsprechung gibt allerdings vor, dass die Grundleistung alleine nicht ausreichend ist, um fahrgeeignet zu sein, weil Leistungsreserve notwendig ist, um auf aussergewöhnliche Ereignisse zu reagieren, z.B. plötzlich auftretende, schwierige Verkehrs-, Strassen- und Umweltverhältnisse. Vereinfacht gilt deshalb, dass also je höher das medizinische Grundrisiko ist (z.B. leichte kognitive Beeinträchtigung plus leichte Sehschwäche), desto kleiner ist die verbleibende Leistungsreserve (Abb. 1)

Tab. 1: Die wesentlichen rechtlichen Rahmenbedingungen für die Einschätzung der medizinischen Mindestanforderungen in Memory Kliniken.

\begin{tabular}{|c|c|c|}
\hline & Thema & Inhalt \\
\hline Obligationenrecht (OR) & & (http://www.admin.ch/ch/d/sr/c220.html) \\
\hline OR Art. 398 & Ärztliche Sorgfaltspflicht & Regelt die Sorgfaltspflicht bei einfachen Aufträgen. \\
\hline Strassenverkehrsgesetz (SVG) & & (http://www.admin.ch/ch/d/sr/c741_01.html) \\
\hline Art 14, Abs. 4 & Ärztliches Melderecht & $\begin{array}{l}\text { Jeder Arzt kann Personen, die wegen körperlichen oder geistigen Krankheiten } \\
\text { oder Gebrechen oder wegen Süchten zur sicheren Führung von Motorfahr- } \\
\text { zeugen nicht fähig sind, der Aufsichtsbehörde für Ärzte und der für die Ertei- } \\
\text { lung und den Entzug des Führerausweises zuständigen Behörde melden. }\end{array}$ \\
\hline Verkehrszulassungsverordnung (VZV) & & (http://www.admin.ch/ch/d/sr/c741_51.html) \\
\hline Art. 27 , Abs. $1 \mathrm{~b}$ und $2 \mathrm{a}$ & $\begin{array}{l}\text { Vertrauensärztliche } \\
\text { Kontrolluntersuchung }\end{array}$ & $\begin{array}{l}\text { Gemäss Art. 27. Abs. 1, Buchstabe b häben sich alle Fahrzeugführer, die } \\
\text { den Führerausweis der 3. Gruppe besitzen (z.B. insbesondere Kat. B-Per- } \\
\text { sonenwagen, Kat. A-Motorräder oder landwirtschaftliche Fahrzeuge) ab } \\
\text { dem } 70 \text {. Altersjahr alle zwei Jahre einer medizinischen Kontrollunter- } \\
\text { suchung zu unterziehen, um verkehrsrelevante Erkrankungen auszu- } \\
\text { schliessen. Art. } 27 \text { Abs. 2a regelt, dass diese vertrauensärztlichen Kontroll- } \\
\text { untersuchungen an die behandelnden Ärzte übertragen werden können. }\end{array}$ \\
\hline Art. 29 & Kontrollfahrt & $\begin{array}{l}\text { Dieser Artikel regelt die Kontrollfahrt, die sich für Senioren eignet, die die } \\
\text { medizinischen Mindestanforderungen erfüllen und trotzdem Bedenken über } \\
\text { die Eignung eines Fahrzeugführers bestehen. }\end{array}$ \\
\hline Anhang 1 & $\begin{array}{l}\text { Medizinische } \\
\text { Mindestanforderungen }\end{array}$ & $\begin{array}{l}\text { Dieser Anhang regelt die medizinischen Mindestanforderungen. Für die } \\
\text { Memory Clinics sind die Minimalanforderungen betreffend Nerven- } \\
\text { system (also insbesondere keine schweren Nervenkrankheiten, keine } \\
\text { Geisteskrankheiten von Bedeutung; keine periodischen Bewusstseins- } \\
\text { trübungen oder -verluste; keine Gleichgewichtsstörungen) und Sehen } \\
\text { (für Beidäugige: Visus korrigiert minimal 0,6; Gesichtsfeld minimal } \\
140 \text { Grad, keine Doppelbilder) wichtig. }\end{array}$ \\
\hline
\end{tabular}


(siehe auch Entscheidung des Schweizerischen Bundesgerichts, amtliche Sammlung (BGE) 130 IV 32, Erwägung 3.1).

Patienten, die die medizinischen Mindestanforderungen nicht erfüllen, dürfen nicht zur Kontrollfahrt angemeldet werden. Die Rahmenbedingungen für die Kontrollfahrt sind in der VZV (Art. 29) geregelt. Sie dauert in der Regel 45-60 Minuten und wird im eigenen Fahrzeug durchgeführt. Bei Nichtbestehen kann sie in der Schweiz, anders als in anderen europäischen Ländern, nicht wiederholt werden. Für den Patienten ist es deshalb empfehlenswert, vor der Kontrollfahrt Fahrstunden bei einem konzessionierten Fahrlehrer zu nehmen. In einigen Kantonen (z.B. Zürich, Schaffhausen, Thurgau, Zug, St. Gallen, Solothurn, Luzern und Graubünden) werden im Rahmen von verkehrsmedizinischen Abklärungen ärztlich begleitete Kontrollfahrten durchgeführt. Während diesen beurteilt der Experte die Fahrkompetenz und der Arzt die Auswirkungen der Erkrankungen auf das Fahrverhalten. Die Kontrollfahrt eignet sich für Senioren, welche die medizinischen Mindestanforderungen möglicherweise erfüllen und bei denen Bedenken zur Fahreignung trotz Untersuchung nicht ausgeräumt werden können. Typische Indikationen für Kontrollfahrten sind z.B. leichte Demenz; schwierig zu beurteilende kognitive Beeinträchtigung bei fehlender Schulbildung oder Fremdsprachigkeit; Multimorbidität durch das Zusammenwirken von verschiedenen leichten Erkrankungen, die in ihrer Gesamtheit eingeschätzt werden müssen.

Bei leichter kognitiver Beeinträchtigung, einer beginnenden Demenz oder anderen progredienten Erkrankungen ist es sinnvoll, die Kontrollfrist zu verkürzen. Die Fahrtauglichkeit kann aus medizinischen Gründen örtlich, zeitlich oder bezüglich Fahrzeugkategorie (z.B. Kategorie F, $45 \mathrm{~km} / \mathrm{h}$ ) eingeschränkt werden oder es können Auflagen (z.B. Brillentragen) für das Autofahren gemacht

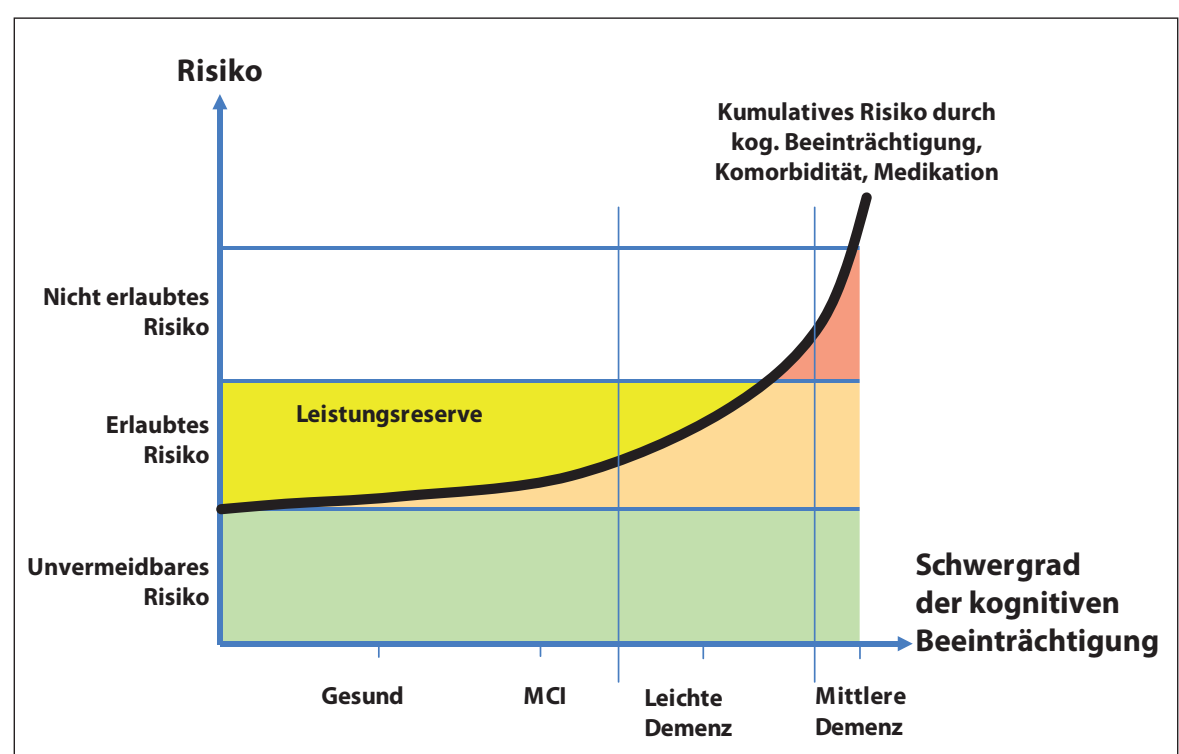

Abb. 1: Schematische Darstellung der Assoziation zwischen Risiko und Leistungsreserve. Je höher das kumulative Risiko (z.B. durch kognitive Beeinträchtigung, Komorbidität und Medikation), desto kleiner die Leistungsreserve.

werden. Bei Demenzen sind die Einschränkungen, z.B. Verminderung des Fahrradius, in der Regel keine Lösung, da diese Limitationen vergessen werden und erfahrungsgemäss viele Schwierigkeiten auch innerorts, also im Bereich von $50 \mathrm{~km} / \mathrm{h}$, auftreten können [6].

\section{Kognition und Autofahren}

Die Kognition ist neben der Motorik und der Perzeption eine wichtige Teilfunktion beim Autofahren. Die kognitive Testung ist ein integraler Bestandteil der Demenzdiagnostik. In den Schweizer Memory Clinics wird als Basismodul für die kognitive Testung oft die Neuropsychologische Testbatterie des Consortium to Establish a Registry for Alzheimer's Disease (CERADPlus; siehe www.memoryclinic.ch) [12] verwendet. In der Literatur [3] werden für das Autofahren neben der kognitiven Gesamtleistung die Aufmerksamkeit, die exekutiven und visuellen Funktionen sowie das Reaktionsvermögen als besonders relevant beurteilt.

Die Aufmerksamkeit ist eine basale kognitive Leistung und entscheidend für die Informationsselektion (d.h. selektive oder fokussierte Aufmerksamkeit) und Verarbeitung, z.B. gleichzeitige Verarbeitung von visuellen und akustischen Signalen (geteilte Aufmerksamkeit) [3]. Aufmerksamkeit und exekutive Funktionen sind eng miteinander verknüpft. Letztere umfassen Planung, Antizipation von Veränderungen, Umstellfähigkeit, Flexibilität, Antrieb, Motivation und Impulskontrolle. Visuelle Perzeption ist für das Erkennen von Gefahrensituationen, von Verkehrsschildern, für den Überblick und die Orientierung im Verkehr sehr entscheidend. Bei der Testung der visuellen Perzeption werden ein normales Gesichtsfeld, normales Kontrastsehen und gute Sehschärfe vorausgesetzt. All diese Funktionen sind für den Verkehr relevant, da die Beeinträchtigungen zu Fehlwahrnehmungen oder Fehleinschätzungen führen können.

In der CERAD-Plus neuropsychologischen Testbatterie wird visuelle Perzeption unter anderem im Boston Naming Test und in den visuo-konstruktiven Teilaufgaben geprüft. Die Aufmerksamkeits- und exekutiven Funktionen wer- 
den im Trail Making Test untersucht [13]. Mehrere Studien haben die Assoziation zwischen Trail Making Test und Fahrsicherheit untersucht [14,15]. In einer Meta-Analyse war der Trail Making B Test einer der besten Prädiktoren für das Bestehen einer praktischen Fahrprobe [14]. Der Trail Making Test wird zusammen mit anderen Testverfahren (z.B. Uhrentest) von der American Medical Association als Bestandteil für die kognitive Screening-Untersuchung für die Beurteilung der Fahreignung bei Senioren empfohlen [11]. Aufmerksamkeitsdefizite sind jedoch diagnostisch unspezifisch. Sie bestehen bei neurodegenerativen Erkrankungen, bei psychischen Störungen (z.B. Depression) oder sie sind Nebenwirkungen von psychotropen Substanzen (z.B. Benzodiazepinen). Die Mini-Mental-Status Examination (MMSE) [16] und der Uhrentest sind häufig verwendete kognitive Screening-Verfahren, die jedoch wenig mit der Fahrleistung korrelieren [17]. Sie wurden nicht für die Fahreignungstestung entwickelt, wodurch ihre Aussagekraft bei der Beurteilung der Fahreignung begrenzt ist.

Im Hinblick auf Verkehrssicherheit soll neben der Gesamtleistung ein besonderes Augenmerk auf die exekutiven und visuell perzeptiven Funktionen geworfen werden (d.h. CERAD Gesamtleistung; MMSE; Scores in Boston Naming Test, visuo-konstruktive Aufgaben, Trail Making Test). Die Richtlinien der American Academy of Neurology (AAN) weisen darauf hin, dass die Fahrleistung bei MMSE-Werten $\leq 24 / 30$ und Trail Making Test, Part B-Werte $>180$ s nachlässt [9]. Als Faustregel gilt in der verkehrsmedizinischen Praxis, dass ältere Fahrzeuglenker mit einem MMSE $<21$ oder einem pathologischen Trail Making Test Part-A (d.h. mit Fehlern und Zeitüberschreitung [ $>180 \mathrm{~s}]$ ), eine Kontrollfahrt nicht mehr bestehen [6]. Obwohl solche Testwerte, wenn sie isoliert betrachtet werden, nicht genügend aussagekräftig sind, können sie in der Praxis als Bestandteil der Risikobeurteilung hilfreich sein. Wenn die Testleistung über den genannten Schwellenwerten liegt, kann nicht zwingend gefolgert werden, dass die medizinischen Mindestleistungen erhalten sind, da dafür die Demenzätiologie, die Komorbiditäten und die Medikation berücksichtigt werden müssen.

\section{Andere kognitive Screening- Verfahren}

Für die vertiefte Beurteilung von verkehrsrelevanten kognitiven Funktionen werden in einigen Memory Clinics zusätzliche Testverfahren eingesetzt. Beispiele sind das Wiener Test-System (Schuhfried ${ }^{\circledR}$; www.schuhfried.at), die Testbatterie ART 2020 [18], der Test zur Aufmerksamkeitsprüfung (TAP-Mobilität, www.psytest.de) [19], oder der Useful Field of View Test (UFOV) [20]. Diese Computer unterstützten Testverfahren haben den Vorteil, dass sie u.a. Reaktionszeiten alters-, geschlechts- und ausbildungsadaptiert erfassen können.

Es sind uns keine Studien bekannt, welche die Zusammenhänge zwischen der CERAD-Plus Leistung und Fahreignung untersuchen. Beim UFOV Test bestehen gute Korrelationen zwischen Testleistung und Fahrleistung bei Senioren ohne Demenz [21]. Es fehlen Studien, welche die Zusammenhänge zwischen spezialisierten Testverfahren und Fahrsicherheit bei Demenzen untersuchen. Deshalb ist der Mehrwert der spezialisierten Testung bei Patienten mit leichter Demenz noch ungewiss $[9,10]$. Wiederholt geäusserte Kritikpunkte betreffen die Testfairness, die Augenscheinvalidität und der Umstand, dass die Zusammenhänge zwischen Kognition und Motorik der Extremitäten schlecht untersucht werden.

\section{Die leichte kognitive Beeinträchtigung}

Nur zwei Studien haben die Fahrleistung bei leichter kognitiver Beeinträchtigung (engl. mild Cognitive Impairment [MCI]) untersucht [22,23]. Eine Studie [22] fand eine leichte Verunsicherung beim Manövrieren bei Probanden mit MCI. Die andere Studie [23] verglich die Fahrfähigkeit im Fahrsimulator bei Probanden mit MCI und Patienten mit leichter Alzheimer-Erkrankung. Sie fand im Vergleich zu Kontrollen bei Gesunden nur minimale Beeinträchtigungen bei einer Kontrollfahrt im Fahrsimulator. Aufgrund der klinischen Erfahrung und der publizierten Evidenz können Senioren mit MCI die medizinischen Mindestanforderungen in der Regel erfüllen. Ausnahmen bestehen bei MCI-Erkrankten mit prominenten exekutiven Störungen (v.a. beginnende frontale Demenz) und bei MCI-Erkrankten mit anderen verkehrsrelevanten Komorbiditäten. Da ein grosser Teil der MCI-Patienten in der Folge eine Demenzerkrankung entwickelt, sind jährliche Verlaufskontrollen sinnvoll.

\section{Demenz}

Es fehlt ein Goldstandard, um die Auswirkungen einer Demenz für den Strassenverkehr zu beurteilen. Die AAN hat für Demenz-Erkrankte mit leichter Krankheitsausprägung (Clinical Dementia Rating [CDR] 0,5-1) [24] Risikomerkmale beim Autofahren definiert [9]. $\mathrm{Zu}$ diesen gehören die Einschätzung der Angehörigen, dass der Erkrankte grenzwertig oder unsicher fährt; eine Vorgeschichte mit Unfällen oder Bussen; die selbstgewählte Einschränkung der Fahrleistung oder Fahrexposition; eine MMSEPunktezahl $\leq 24$ und aggressive oder impulsive Persönlichkeitsmerkmale. Zusätzliche Risikofaktoren sind der Einfluss von Alkohol oder psychotropen Substanzen und visuelle oder motorische Einschränkungen.

\section{Schweregrad}

Es ist unbestritten, dass mittelschwer bis schwer Demenzerkrankte nicht mehr Autofahren dürfen [9,25]. Zwei prospektive Studien zeigen klar, dass die Erkrankungsprogression und der Schweregrad 
der Alzheimer-Erkrankung die Fahreignung beeinträchtigen und das Unfallrisiko erhöhen [26,27]. Der Schweregrad wurde in beiden Studien mit der Clinical Dementia Rating (CDR) Skala beurteilt [24]. Die Skala berücksichtigt die Gedächtnisleistung, die Orientierung, die Problemlösungsstrategien, die Alltagsfunktionen und die Selbstversorgung. In der Studie von Ott et al. [27] haben innerhalb von einem Jahr 30\% der an Alzheimer Erkrankten mit CDR 0,5 und 50\% der Erkrankten mit CDR 1,0 auf das Autofahren verzichtet. Die häufigsten Gründe für den Verzicht waren das Fortschreiten der Demenz, das Nichtbestehen einer Fahrprobe oder ein Unfall. Die Mehrheit der Demenz-Erkrankten mit CDR 0,5 (d.h. 67-85\%) oder CDR 1 (41-76\%) bestanden jedoch eine Fahrprobe bei Studieneinschluss [26,28], was eindrücklich illustriert, dass der Zeitpunkt der Diagnose «leichte Demenz» nicht zwingend mit dem Zeitpunkt gleichzusetzen ist, an dem das Autofahren aufgegeben werden muss. Von den vielen Skalen für die Beurteilung des Schweregrades der Demenz ist die CDR-Skala am weitesten verbreitet $[9,29]$. Sie wurde für die Beurteilung der Alzheimer-Demenz entwickelt, und sollte vor allem dort eingesetzt werden. Da die Demenz eine progrediente Erkrankung ist, haben Verlaufsuntersuchungen (z.B. alle 6-12 Monate) eine grosse Bedeutung.

\section{Ätiologie}

\section{Alzheimer-Erkrankung}

Die Zusammenhänge zwischen Autofahren und Demenz sind am besten bei der Alzheimer-Erkrankung untersucht [23,26,27]. Studien zeigten, dass Alzheimer-Kranke beim Autofahren mehr Fehler machen als gesunde Senioren oder Senioren mit MCI [23,30]. Zwei prospektive Studien fanden, dass die Fahreignung bereits in den frühen Krankheitsstadien beeinträchtigt ist $[27,30]$. Die Beeinträchtigungen sind bei Patienten mit CDR $>1$ derart, dass die medizinischen
Mindestanforderungen für das Autofahren i.d.R. nicht mehr erfüllt sind [3].

\section{Fronto-temporale Lobär- degenerationen}

Die Fahreignung bei der Verhaltensvariante der fronto-temporalen Lobärdegenerationen (FTD) (behavioural variant of fronto-temporal dementia) [31] ist in einer kleinen Fahrsimulator-Studie untersucht worden [32]. Die Fahreignung war schwer beeinträchtigt und das Ausmass der Beeinträchtigung korrelierte mit den Verhaltensauffälligkeiten. Initialsymptome dieser Demenzform sind Persönlichkeitsveränderungen, Verhaltensstörungen und exekutive Dysfunktion, die allesamt nicht mit sicherem Autofahren zu vereinbaren sind. Deshalb sind hier die medizinischen Mindestanforderungen in der Regel nicht mehr erfüllt. Schwierig ist die Einschätzung der medizinischen Mindestanforderungen bei den frontotemporalen Demenzformen mit prominenten Sprachstörungen (primär progrediente Aphasie und semantische Demenz) [33], da der Schweregrad der kognitiven Beeinträchtigung wegen der Sprachstörung schwer quantifizierbar ist. Falls die Alltagsfunktionen nicht beeinträchtigt sind und keine anderen Komorbiditäten bestehen, kann die Fahrsicherheit oft nur im Strassenverkehr selbst (d.h. während einer Kontrollfahrt) beurteilt werden.

\section{Demenzen mit extrapyramidal- motorischen Störungen}

Da es beim Autofahren die Integration von Kognition, Perzeption und Motorik braucht, erfüllen Demenz-Erkrankte Patienten mit motorischen und/oder perzeptiven Beeinträchtigungen die medizinischen Mindestanforderungen des VZV in der Regel nicht mehr. Eine Studie untersuchte die Fahreignung bei Patienten mit Huntington-Krankheit [34]. Die Patienten hatten im Vergleich zu gesunden Probanden mehr Kollisionen in der Vorgeschichte und mehr Schwierigkeiten im Fahrsimulator. Mehrere Studien unter- suchten die Fahreignung mit M. Parkinson und zeigten, dass für die Beurteilung der Fahreignung die extrapyramidalmotorischen Störungen, die kognitiven Beeinträchtigungen, die visuell perzeptiven Veränderungen, die Alltagsfunktionen und die Tagesschläfrigkeit mitberücksichtigt werden müssen [35-40]. Da diese Studien Patienten mit Parkinson-Demenz (PDD) ausschlossen, fehlt Studienevidenz zur Fahrsicherheit bei ParkinsonDemenz [41] oder Lewy KörperchenDemenz (dementia with Lewy bodies [DLB]) [42]. Die Hauptmerkmale dieser Demenzformen sind die Fluktuation der Kognition, extrapyramidal-motorische Defizite, Bradyphrenie, perzeptive Beeinträchtigungen und visuelle Halluzinationen, die insgesamt kaum mehr mit Fahreignung zu vereinbaren sind.

\section{Vaskuläre Demenzen}

Obwohl zerebrovaskuläre Erkrankungen eine häufige Ursache für Demenzerkrankungen sind, fehlen Studien, welche die Zusammenhänge zwischen vaskulärer Demenz und Fahreignung untersuchen. Die klinische Manifestation von vaskulären Demenzen hängt vom Ausmass und der Lokalisation der vaskulären Läsionen ab. Deshalb wird es schwierig sein, generalisierbare Regeln zur Fahreignung bei vaskulärer Demenz zu entwickeln. Neben den kognitiven und motorischen Ausfällen sind bei diesen Erkrankungen insbesondere auch Doppelbilder, Gesichtsfeldausfälle, Verlangsamung und visuo-spatialer Neglekt zu berücksichtigen [43].

\section{Demenz-assoziierte Sympto- me, die bei der Beurteilung der medizinischen Mindest- anforderungen berücksich- tigt werden müssen}

\section{Einsicht in die kognitiven Defizite und Kompensationsstrategien}

Es besteht bei den meisten Verkehrsteil- 
nehmern, unabhängig vom Alter, die Tendenz, das eigene Fahrvermögen zu überschätzen. Eine sichere Verkehrsteilnahme ist jedoch nur möglich, wenn Betroffene die kognitive Einschränkung wahrnehmen können. Viele Senioren entwickeln Kompensationsstrategien und vermeiden Risiken durch Routenwahl (nur bekannte Routen), durch die Wahl des Zeitpunktes der Autofahrt (z.B. Vermeiden von Fahrten in der Nacht, bei schlechter Witterung, oder in Stosszeiten) und durch Strategien während der Fahrt (z.B. langsameres Fahren, mehr Abstandkontrolle, Vermeiden von Überholmanövern) [44]. Mit einer fehlenden Selbstwahrnehmung der Krankheit (Anosognosie) können die Folgen der kognitiven Beeinträchtigungen für den Strassenverkehr nicht mehr realistisch beurteilt werden. Das beeinträchtigt den Gebrauch von Kompensationsstrategien [45] und führt zu Fehleinschätzungen im Verkehr [46]. Viele Demenzkranke schränken die Fahrdistanz ein (weniger Kilometer pro Jahr), doch scheint diese Vermeidungsstrategie das Unfallrisiko eher zu erhöhen [9]. Hinweise auf Anosognosie sind eine unterschiedliche Selbst- und Fremdeinschätzung bezüglich der kognitiven Leistungen [47] oder der Alltagsfunktionen [48]. In der Regel können die medizinischen Mindestanforderungen bei Demenz mit Anosognosie nicht mehr erfüllt werden.

\section{Aggressives und impulsives Verhal- ten und andere demenzassoziierte Verhaltensstörungen}

Aggressives und impulsives Verhalten bei Demenzerkrankten kann zu Verkehrsregelverletzungen und Unfällen führen [49]. Diese Symptome können mit dem Neuropsychiatrischen Inventar (NPI) gemessen [50] und müssen bei der Beurteilung der medizinischen Mindestanforderungen kritisch berücksichtigt werden, da sie mit einer Fahreignung nicht zu vereinbaren sind.

\section{Andere wesentliche Komor- biditäten und Medikamente}

Die Komorbiditäten und die medizinischen Mindestanforderungen sind im Anhang $1 \mathrm{VZV}$ beschrieben [51]. Die meisten verkehrsrelevanten Komorbiditäten werden bei der Demenzdiagnostik ohnehin erfasst. Die wichtigsten sind die Folgenden:

\section{Altersassoziierte Sehstörungen}

Sehstörungen nehmen altersabhängig zu. Die häufigsten Ursachen sind Katarakt, diabetische Retinopathie, senile Makuladegeneration, Glaukom, Augenmuskelparesen, supranukleäre Störungen der Okulomotorik und Störung der höheren visuellen Funktion (z.B. Hemianopsie). Falls altersassoziierte Augenerkrankungen bekannt sind oder wenn sich in der klinischen Routineuntersuchung Sehstörungen finden, empfiehlt sich für die Einschätzung der Auswirkungen für den Strassenverkehr und die Prüfung der therapeutischen Möglichkeiten eine spezialärztliche ophthalmologische Untersuchung [52]. Es ist ungewiss, ob durch präventive visuelle Screening-Untersuchungen die Verkehrssicherheit verbessert werden kann [53]. Die medizinischen Mindestanforderungen (VZV Anhang 1) legen fest, dass der korrigierte Fernvisus an einem Auge mindestens 0,6 und am anderen Auge 0,1 sein muss. Falls diese Anforderungen nur mit Brille oder mit Kontaktlinsen erreicht werden können, muss die entsprechende Auflage im Führerausweis vermerkt sein. Die horizontale Ausdehnung des Gesichtsfeldes muss mind. 140 Grad betragen und es dürfen keine Doppelbilder bestehen. Bei Patienten mit Glaukom ist eine fachärztliche Gesichtsfeldprüfung zum Ausschluss eines Zentralskotoms sinnvoll.

\section{Diabetes mellitus}

Die Schweizerische Gesellschaft für Diabetologie hat zusammen mit der Schweizerischen Gesellschaft für Rechtsmedizin
Richtlinien für die Beurteilung der Fahrfähigkeit bei Diabetes mellitus erarbeitet [54]. Die Diabetes mellitus assoziierten Risiken sind einerseits die Bewusstseinsbeeinträchtigung durch Hypoglykämie und anderseits die Spätfolgen der Erkrankung (v.a. diabetische Retinopathie, koronare Herzerkrankungen, periphere Polyneuropathie). Besteht ein Diabetes mellitus bei gleichzeitiger Demenz, können Schwierigkeiten bei der Compliance und der medikamentösen Selbstkontrolle zu Hyper- oder Hypoglykämien führen, was bei der Beurteilung der Fahreignung berücksichtigt werden sollte. Wir verweisen auf die kürzlich publizierten Richtlinien [54,55] (http:// www.diabetesgesellschaft.ch).

\section{Erkrankungen mit Bewusstseins- verlust und Epilepsie}

Senioren sind mittlerweile die grösste Altersgruppe mit Epilepsien und die meisten Betroffenen, d.h. 75\%, bleiben nach erfolgreicher Therapie anfallsfrei. Die Richtlinien der Schweizerischen Liga gegen Epilepsie (www.epi.ch) empfehlen bei einem unprovozierten Anfall sechs Monate Fahrkarenz und bei mehr als einem Anfall zwölf Monate Fahrkarenz [56]. In der Praxis [57] wird bei Synkopen mit Behandlungsmöglichkeiten eine Fahrkarenz bis zur erfolgreich abgeschlossenen Therapie (z.B. Schrittmacherimplantation) empfohlen.

\section{Pathologische Tagesschläfrigkeit}

Die Schweizerische Gesellschaft für Schlafforschung, Schlafmedizin und Chronobiologie hat zum Thema Fahreignung bei Tagesschläfrigkeit Richtlinien publiziert [58], die die Beurteilung und das praktische Vorgehen bei der Fahreignung bei Tagesschläfrigkeit regeln. Die häufigsten Ursachen der Tagesschläfrigkeit im Alter sind die neurodegenerative Erkrankung selbst (vor allem Lewy-KörperchenDemenz und Parkinson-Demenz), das Schlaf-Apnoe-Syndrom, Medikamente (v.a. Benzodiazepine, Antihistaminika, 
Antiepileptika, Antidepressiva, Dopaminagonisten und Opiate) und die Insomnie. Tagesschläfrigkeit kann mit dem Maintenance of Wakefullness Test (MWT) [59] oder dem Epworth-Fragebogen [60] quantifiziert werden. Bei Letzterem sind Summenwerte über 10 abnorm und Werte über 12 korrelieren mit Unfallereignissen [61]. Das Schlaf-ApnoeSyndrom ist i.d.R. therapierbar. Unbehandelt kann dies die vaskulären Risiken erhöhen und zu kognitiven Beeinträchtigungen und depressiven Verstimmungen führen.

\section{Autofahren nach Schlaganfall}

Eine Kontrolluntersuchung zur Überprüfung der medizinischen Mindestanforderungen nach Schlaganfall ist in jedem Fall indiziert. Zerebrovaskuläre Ereignisse erhöhen das Risiko für eine sekundäre demenzielle Entwicklung und sie können die Erstmanifestation einer neurodegenerativen Erkrankung sein. Bei der Beurteilung nach Schlaganfall sollten Kognition, vaskuläre Risikofaktoren, motorische Defizite, kognitive Ausfälle, hemispatialer Neglekt und Hinweise auf Augenmotilitätsstörungen berücksichtigt werden [43]. Einige Defizite sind innerhalb der ersten Monate nach Schlaganfall regredient, weshalb eine freiwillige Fahrpause von 3-6 Monaten vor der Beurteilung der Fahreignung vorteilhaft sein kann.

\section{Psychische Erkrankung}

Psychiatrische Komorbiditäten, insbesondere im Kontext von neurodegenerativen Erkrankungen, müssen bei der Beurteilung der Fahreignung berücksichtigt werden. Am häufigsten sind affektive Störungen (z.B. Angst, Depression), Suchterkrankungen (bei Senioren insbesondere Benzodiazepine und Alkohol), die abhängig vom Schweregrad der Erkrankung oder der Therapie die Verkehrssicherheit beinträchtigen $[62,63]$. Wenn die psychische Erkrankung im Kontext einer Demenz besteht, ist für die Beurteilung der Fahreignung in jedem Fall eine vertiefte Abklärung notwendig.

\section{Psychotrope Medikamente}

Die Liste der psychotropen Substanzen, welche die Fahrfähigkeit (und evtl. auch die Fahreignung) beeinflussen können, ist lang. Die Risiken sind am grössten in den ersten Wochen der Therapie und bei Dosisanpassung. Im Alter steigt das Risiko der Dosisakkumulation bei Substanzen mit langer Halbwertszeit, weshalb der Zeitpunkt der Medikamenteneinnahme und die Polypharmazie berücksichtigt werden müssen. Die wichtigsten Substanzen, die die Verkehrssicherheit beeinflussen, umfassen Benzodiazepine [64]; Benzodiazepinähnliche Substanzen (Zolpidem, Zopiclon und Zaleplon); Substanzen mit anticholinergen (z.B. trizyklische Antidepressiva), antihistaminergen Wirkungen, Insulin und Sulfonylharnstoffe (Hypoglykämiegefahr), Dopaminagonisten (zur Therapie des Morbus Parkinson) bzw. -Antagonisten (Antipsychotika) und zentral wirkende Schmerzmittel. Senioren, die tagsüber Benzodiazepine nehmen, sollten nicht mehr autofahren. Wenn diese Substanzen verschrieben werden, müssen die Auswirkungen auf die Fahrfähigkeit erwähnt werden. Zu Beginn der Therapie oder bei einer Dosiserhöhung sollte eine Fahrpause während 1-2 Wochen erwogen werden.

Das International Council on Alcohol, Drugs and Traffic Safety (ICADTS) hat eine dreistufige Kategorisierung für psychotrope Substanzen publiziert [65] (http://www.icadts.nl/medicinal.html).

Die Kategorisierung basiert auf Blutalkohol-Äquivalenzdosen. Medikamente der Kategorie I haben keinen signifikanten Einfluss auf die Verkehrssicherheit (Äquivalenzdosis zu Blutalkoholgehalt $<0,5$ Promille) (z.B. Fluoxetin, Paroxetin); solche der Kategorie II haben einen kleinen Effekt auf die Verkehrssicherheit (Äquivalenzdosis zu Blutalkoholgehalt
0,5-0,8 Promille) (z.B. Imipramin, Nortriptylin) und jene der Kategorie III sind Substanzen mit Gefahrenpotenzial, da sie die Verkehrssicherheit beeinträchtigen (Äquivalenzdosis zu Blutalkoholgehalt $>0,8$ Promille) (z.B. Benzodiazepine, Anxiolytika) (http://www.icadts.nl/ medicinal.html) [65]. Bei den Antidepressiva beeinträchtigen Substanzen mit sedierenden Nebenwirkungen (Mirtazapin, Trazodon) die Verkehrssicherheit mehr als solche ohne Sedation (z.B.SSRI). Bei den Antipsychotika führen Atypika zu etwas weniger Fahrbeeinträchtigungen als z.B. Haloperidol [66]. Die Wirkung der psychotropen Substanzen wird durch Polypharmazie und Alkohol verstärkt [67]. Es fehlen Studien, die bei Patienten mit leichter Demenz die Auswirkungen von psychotropen Substanzen auf die Fahreignung untersuchen.

\section{Empfehlungen zur Beurtei- lung der Fahreignung bei kognitiver Beeinträchtigung bzw. Demenz}

Bei jeder Abklärung sollte erhoben werden, ob der Patient ein Motorfahrzeuglenker ist und wie seine Zukunftsplanung beim Autofahren aussieht. Für die Beurteilung der verkehrsrelevanten Auswirkungen einer Demenz muss neben dem Mobilitätsbedürfnis, auch das Risiko durch die kumulative Komorbidität berücksichtigt werden. Die Demenzursache und der Schweregrad der Erkrankung sowie die verkehrsrelevanten Komorbiditäten werden im diagnostischen Prozess in der Memory Clinic ohnehin erfasst und beurteilt [1]. Da die Kernkompetenz der Memory Clinic die Demenzdiagnostik ist, gelten für die Berücksichtigung der Komorbiditäten ähnliche Anforderungen wie für die gesetzlich vorgeschriebene periodische Kontrolluntersuchung, die in der Regel von den Hausärzten durchgeführt wird [51,57]. Bei Unklarheiten empfiehlt sich die Zusammenarbeit mit den Spezialisten. Die Anhänge 2 und 3 der VZV [68] 


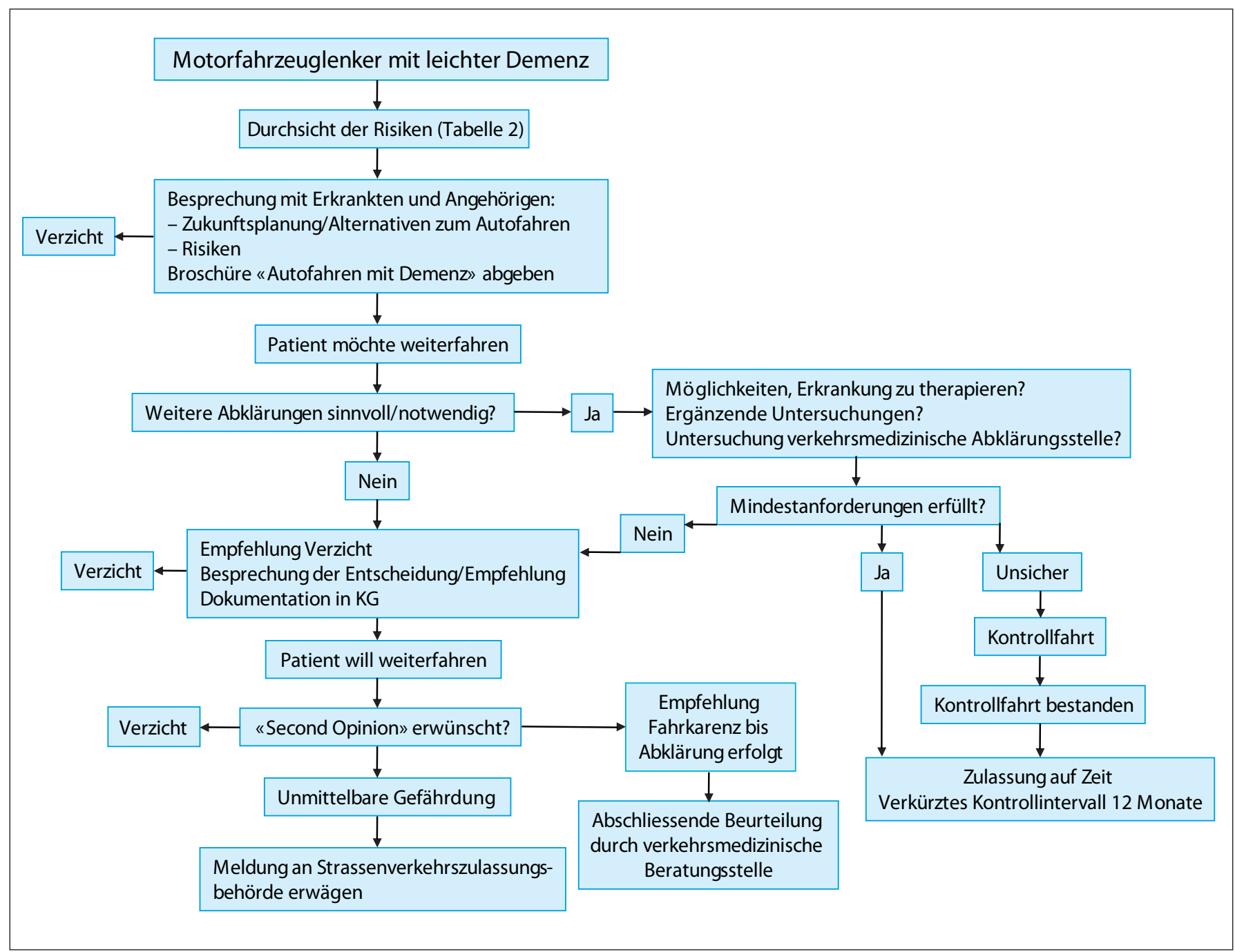

Abb. 2: Zeigt einen möglichen Abklärungsentscheidungsprozess für die Fahrberatung von Senioren mit leichter Demenz.

enthalten Entwürfe für ärztliche Zeugnisse oder für medizinische Begutachtung.

$\mathrm{Zu}$ Beginn der Beratung werden von den Betroffenen oft die unfallfreien Jahre betont. Es hilft zu vermitteln, dass es hier keinesfalls um die Beurteilung "guter oder schlechter Autofahrer geht», sondern um die Einschätzung, ob die medizinischen Mindestanforderungen für die Zulassung zum Strassenverkehr trotz kognitiver Beeinträchtigung noch erfüllt sind. Sobald die Erkrankten über die Diagnose und die Krankheitsprogression aufgeklärt sind und verstehen, dass die Fahreignung durch Demenz in jedem Fall infrage gestellt wird und das bestmögliche Ergebnis einer vertieften und kostspieligen Fahreignungsabklärung allenfalls die Erhaltung der Fahreignung um ein weiteres Jahr beträgt, zeigen sich die meisten Patienten einsichtig. Das Ziel der Beratung, welche die Besprechung der Sicherheit und der Alternativen einschliesst, sollte der freiwillige Verzicht auf das Autofahren sein. Ein mögliches Entscheidungsschema ist in Abbildung 2 wiedergegeben.

Falls ein Motorfahrzeuglenker mit leichter Demenz weiter Autofahren möchte, empfiehlt es sich, fremd- und eigenanamnestisch gezielt nach Schwierigkeiten (Polizeikontrollen, Verkehrsbussen, FastUnfälle, Unfälle oder Parkschäden) oder
Unsicherheiten (z.B. Verfahren in gewohnter Umgebung, Unsicherheitsgefühl bei Fahrer oder Beifahrer) während der letzten beiden Jahre zu fragen. Es interessiert auch, ob sich das Fahrverhalten verändert hat (z.B. keine Fahrten in der Nacht, bei dichtem Verkehr, bei schlechter Witterung) und wie viele Kilometer pro Jahr gefahren werden. Die Fremdanamnese ist sehr wichtig, da Ereignisse vergessen werden und bei allen Lenkern, ob jung oder alt, die Neigung besteht, das eigene Fahrvermögen zu überschätzen. Stark divergierende Angaben geben möglicherweise Hinweise auf fehlende Krankheitswahrnehmung. Die Angaben müssen sorgfältig dokumentiert werden, auch wenn Schwierigkeiten verneint 
werden. Die Canadian Medical Association [69] (http://www.cma.ca/index.php/ ci_id/18223/la_id/1.htm) und die American Medical Association [11] (http:// www.ama-assn.org/ama/pub/physicianresources/public-health/promoting-healthy-lifestyles/geriatric-health/older-driversafety/assessing-counseling-older-drivers. page) haben umfangreiche Handbücher für die Beurteilung der Fahreignung entwickelt. In diesen sind auch Vorschläge zur Verkehrsanamnese enthalten und in einigen wenigen Memory Clinics in der Schweiz werden Übersetzungen verwendet. Autofahren kann zum Konfliktthema zwischen Patienten und Angehörigen werden, vor allem wenn die Krankheitseinsicht fehlt. Angehörige können bei ihren Einschätzungen aus Mitgefühl oder Scheu vor Konflikten befangen sein. Sie sind von der Einschränkung der Mobilität mitbetroffen und müssen deshalb wenn immer möglich entlastet werden. Die Alzheimervereinigung hat eine hilfreiche Informationsbroschüre für Patienten und Angehörige erarbeitet, die im Web einfach zugänglich ist (Publikation: Autofahren und Demenz; (C2010 Schweizerische Alzheimervereinigung http:// www.alz.ch/d/html/publikationen.html)

Im Somato- und Neurostatus in der Memory Clinic sind in der Regel nahezu alle Befunde für eine komplette verkehrsmedizinische Beurteilung vorhanden. Die Ausnahmen sind die Prüfung des Sehens und die Prüfung der Halswirbelsäulenbeweglichkeit. Bei Letzterer muss auf eine genügende Auslenkung der Augenebene (rascher Seitenblick mindestens 45 Grad beidseits) geachtet werden. Das Sehen wird in der Memory Clinic oft nur marginal untersucht. Deshalb braucht es Ergänzungen, die selbst oder in Zusammenarbeit mit einem Ophthalmologen durchgeführt werden können. Bei der Untersuchung der Augen müssen die Augenbewegungen (Ausschluss Doppelbilder und Blickparesen) und das Gesichtsfeld durch konfrontative Testung und der Fernvisus (korrigiert und unkorrigiert) unter opti-
Tab. 2: Risikoeinschätzung für die Beurteilung der medizinischen Mindestanforderungen bei beginnender Demenz.$$
\text { Risikoeinschätzung }
$$

\section{Anamnese}

Polizeikontrollen, Verkehrsbussen, Unfälle (letzte 2 Jahre)

Beifahrer fühlt sich unsicher ...

Vermeidungsstrategien - Einschränkung Fahrleistung

Clinical Dementia Rating (CDR)

CDR: 0,5

CDR: 1,0 .......

CDR: $>1,0$

\section{Alltagsaktivitäten}

Leichte Beeinträchtigung IADL

Beeinträchtigung BADL

\section{Mini Mentalstatus}

MMSE $>24$

MMSE 22-24

MMSE $\leq 21$.

\section{Trail Making B Test}

Testwert $<180 \mathrm{~s}$

Testwert $>180-200 \mathrm{~s}$

Testwert $>300$ (d.h. nicht mehr durchführbar)

\section{Sehen}

Fernvisus >0,6; Gesichtsfeld >140 Grad; keine Doppelbilder,

kein Neglekt...

Fernvisus $<0,6$

Gesichtsfeld $<140$ Grad

Doppelbilder

Hemispatialer Neglect

\section{HWS-Beweglichkeit}

Kopfdrehen $>45$ Grad

Kopfdrehen $<45$ Grad

\section{Demenzätiologie und Schweregrad}

Sehr leichte Alzheimer-Demenz (CDR 0,5; MMSE $\geq 24$, Trail B <180) ...... Leichte Alzheimer-Demenz (CDR 1, MMSE $\geq 24$, Trail B <180).

Frontotemporale Degeneration (Verhaltensvariante)

Lewy Körperchen-Demenz (MMSE $\geq 24$, Trail B $<180$ )

Parkinson-Demenz (MMSE $\geq 24$, Trail B $<180$ )

\section{+ zusätzliche demenzassoziierte Komorbiditäten}

Impulsiv, aggressiv (z.B. neuropsychiatrisches Interview)

Fehlende Krankheitseinsicht

Tagesschläfrigkeit (Epworth Fragebogen $\geq 12$ )

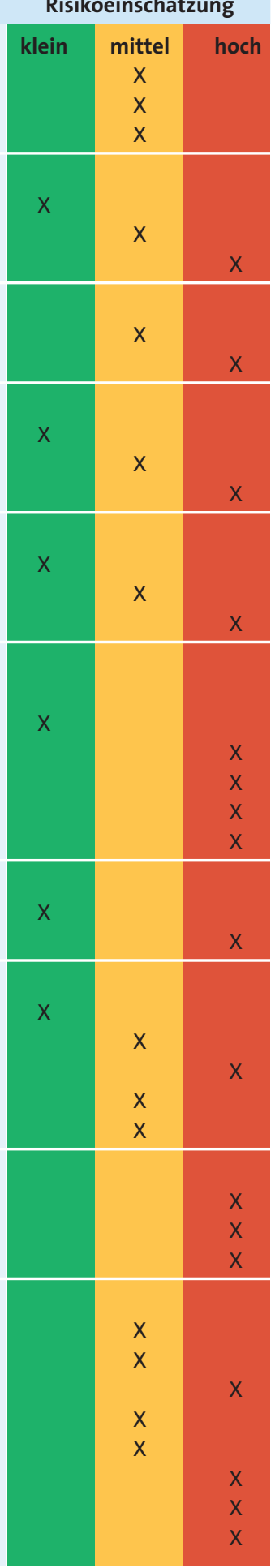

\section{+ andere verkehrsrelevante Komorbiditäten}

Psychotrope Substanzen (ICADTS Stufe $\geq 2$ ) (Dauertherapie)

Polypharmazie mit psychotropen Substanzen (Dauertherapie)

Hinweise auf Suchterkrankung (Benzodiazepine, Alkohol)

Diabetes mellitus mit körperlichen Spätfolgen*..

Diabetes mellitus mit Hypoglykämie*.....

Epilepsie (asymptomatisch $<12$ Monate)**

Synkope (asymptomatisch $<6$ Monate) .

Zerebrovaskulärer Insult $<6$ Monate

* siehe Richtlinien der Schweizerischen Gesellschaft für Diabetologie

${ }^{* *}$ siehe Richtlinien der Schweizerischen Liga gegen Epilepsie

Patienten mit hohen Risiken erfüllen die medizinischen Mindestanforderungen i.d.R. nicht mehr. Sie sollten nicht zur Kontrollfahrt zugelassen werden. Bei Patienten mit Risiken im mittleren, gelben Bereich muss das kumulative Risiko beurteilt werden und Möglichkeiten zur Minimierung der Risiken durch Therapie geprüft werden. Allenfalls kann eine Kontrollfahrt Klarheit schaffen. 
malen Lichtverhältnissen für jedes Auge einzeln geprüft werden [57].

In Tabelle 2 sind die wesentlichen Risiken zusammengefasst und im Konsensus wurden die Risiken in kleine (grün), mittlere (gelb) und grosse Risiken (rot) eingeteilt. Diese Einteilung kann bei der individuellen Risikoeinschätzung helfen, obwohl es nicht möglich ist, das Gesamtrisiko zu quantifizieren. In der Regel sind die medizinischen Mindestanforderungen im roten Bereich nicht mehr erfüllt. Wenn solche Erkrankungen oder Einschränkungen gefunden werden, sollten diese mit dem Patienten und den Angehörigen besprochen werden. Es muss in diesem Fall eine begründete Empfehlung zum Verzicht besprochen und auch erklärt werden, wieso weitere Abklärungen innerhalb der Memory Clinic nicht sinnvoll sind. Falls die Betroffenen eine spezialisierte Zweitmeinung wünschen, ist dies sicherlich in einer verkehrsmedizinischen Abklärungsstelle möglich, doch müssen die Kostenfolgen von den Betroffenen getragen werden. Um eine unnötige und kostspielige Wiederholung von Untersuchungen $\mathrm{zu}$ vermeiden, empfiehlt es sich, dem Patienten den Schlussbericht der Memory Clinic mitzugeben, damit er diesen bei der verkehrsmedizinischen Untersuchung vorlegen kann. Bei Uneinsichtigkeit und wenn Gefahr im Verzug ist, sollte eine Meldung an die Strassenverkehrszulassungsbehörde erwogen werden. Dazu ist keine Entbindung vom Berufsgeheimnis notwendig, da der Gesetzgeber ein Melderecht vorsieht. In einigen Memory Clinics wird vor einer Meldung das Ergebnis der Beurteilung und die Empfehlung des freiwilligen Verzichtes dem Patienten noch schriftlich mitgeteilt. Falls das Melderecht in Anspruch genommen wird, hilft es, die Entscheidung mit dem zuweisenden Arzt abzusprechen. Falls die Entscheidung zum Verzicht auf das Autofahren von den Angehörigen mitgetragen wird, kann möglicherweise der Zugang zum Auto eingeschränkt werden (z.B. Abhängen der Batterie; Verkauf des Autos u.a.).
Wenn keine grossen Risiken gefunden werden und der Betroffene weiter Autofahren möchte, muss das kumulative Risiko unter der Berücksichtigung der kleinen und mittleren Risiken besprochen werden. Allenfalls sind zusätzliche Abklärungen notwendig oder es bestehen Möglichkeiten, gewisse Risiken durch Therapie zu reduzieren (z.B. KataraktOperation). Gewisse Memory Clinics führen zusätzliche verkehrspsychologische Testungen durch. Die Evidenz, dass diese bei diagnostizierter Demenz zur Voraussage der Fahrsicherheit beitragen, fehlt jedoch noch. Falls es nicht möglich ist, die Risiken abschliessend zu beurteilen und die Fahreignung nicht ausgeschlossen ist, kann eine verkehrsmedizinische Untersuchung z.B. mit spezialärztlich begleiteter Kontrollfahrt Klarheit schaffen. In jedem Fall sollte bei Demenz eine Verlaufsuntersuchung in sechs bis max. zwölf Monaten eingeplant werden, damit die Auswirkungen der Krankheitsprogression beurteilt werden können. Die Konsensusempfehlungen sind in der Tabelle 3 zusammengefasst.

Tab. 3: Konsensusempfehlungen (Learningpoints).

\footnotetext{
- Das Memory Kliniken-Team sollte

- im Rahmen des diagnostischen Prozesses erfassen, ob ein(e) Patient/in Motorfahrzeuglenkerin ist.

- die Angehörigen in den diagnostischen Prozess einbeziehen, da die Beurteilung der Fahrsicherheit von Dritten von Bedeutung ist.

- Bei einer von der Verkehrszulassungsbehörde angeordneten Untersuchung in einer Memory Klinik ist es zwingend, dem Exploranden das Auftragsverhältnis, die Kostenfolgen und Verpflichtung der Informationsweitergabe zu erklären.

- Die Diagnose Demenz alleine reicht nicht aus, um die Fahreignung aus medizinischen Gründen zu verneinen. Die Diagnose Demenz sollte zum Anlass genommen werden, um über die Zukunftspläne beim Autofahren zu sprechen. Demenzen sind progrediente Erkrankungen, die im Verlauf zu schlechterer Fahrleistung führen und das Unfallrisiko erhöhen. Eine individualisierte Beratung über die Diagnose und deren Auswirkungen im Strassenverkehr ist sinnvoll, da die meisten Demenzkranken freiwillig auf das Autofahren verzichten. In der Beratung sollten Alternativen besprochen werden.

- Bei der Beurteilung, ob bei einer beginnenden Demenz die medizinischen Mindestanforderungen erfüllt bleiben, muss

- die Ursache und der Schweregrad der Demenz berücksichtigt werden, denn Patienten mit mittelschwerer oder schwerer Demenz erfüllen die Voraussetzungen für das sichere Lenken von Motorfahrzeugen nicht mehr.

- die kumulative Morbidität und die Polypharmazie bei der Entscheidung mitberücksichtigt werden. Eine Gewichtung der Risiken kann für eine Entscheidung sehr hilfreich sein, denn je grösser das medizinische Grundrisiko ist, desto kleiner sind die Leistungsreserven.

die Entscheidung im Konsens, interdisziplinär, anlässlich der Diagnosekonferenz getroffen werden und sie muss im Verlauf nach sechs bis zwölf Monaten überprüft werden.

- Es gibt keinen Goldstandard für die Abklärung und Beurteilung der Fahreignung bei Demenz. Bei Unsicherheiten kann eine zusätzliche verkehrsmedizinische Untersuchung und/oder eine Kontrollfahrt sinnvoll sein. Letztere darf nur angeordnet werden, wenn die medizinischen Mindestanforderungen möglicherweise erfüllt sind, also keine Kontraindikationen für das Autofahren bestehen.

- Wenn bei einer Demenz die Krankheitseinsicht fehlt und der Betroffene nicht freiwillig auf das Autofahren verzichtet, liegt es im Ermessen des behandelnden Arztes/der behandelnden Ärztin, eine Meldung an das Strassenverkehrsamt zu machen. Um dieses Melderecht wahrzunehmen, ist keine Entbindung vom Berufsgeheimnis notwendig. Es empfiehlt sich, vor einer Meldung dem Patienten schriftlich die Einschätzung zur Fahreignung mitzuteilen und Rücksprache mit dem zuweisenden Hausarzt zu nehmen. Das Melderecht soll nicht delegiert werden.
} 


\section{Zukünftige Entwicklungen}

Viele Fragen bleiben bei der Beurteilung der Fahreignung offen. Der Abklärungsprozess sollte weiter vereinheitlicht und prospektiv evaluiert werden. Es ist erstaunlich, wie wenig in bisherigen Studien die Kompensationsstrategien und die Möglichkeiten der Sicherheitsprävention durch Reduktion des kumulativen Risikos (z.B. Autofahren mit abgeschaltetem Mobiltelefon, ohne Alkohol und nur tagsüber) untersucht wurden. Fahrsimulatoren sind zurzeit noch nicht geeignet, um die Fahreignung bei Senioren mit kognitiver Beeinträchtigung zu beurteilen. Sie haben unspezifische Nebenwirkungen (Schwindel, Übelkeit), die Szenarien sind nicht standardisiert und die Untersuchungen, welche für den Schweizer Strassenverkehr die Leistungen im Simulator mit Leistungen im Strassenverkehr vergleichen, fehlen. Mit der Ausbildung von Verkehrsmedizinern wird die Untersuchung und Beurteilung der Fahreignung weiter spezialisiert werden, was den Wissenstransfer zwischen Verkehrsmedizin und anderen medizinischen Disziplinen fördern wird. In prospektiven klinischen Studien, die die Fahreignung bei Senioren mit kognitiver Beeinträchtigung untersuchen, müssen die noch offenen Fragestellungen geklärt werden. Wir hoffen, dass wir durch diese Zusammenstellung des aktuellen Wissens einen Beitrag zur Vernetzung des Wissens zwischen verschiedenen Disziplinen und zwischen Therapeuten, Betroffenen und Angehörigen leisten können.

\section{Dank}

Die Arbeit der Konsensus-Expertengruppe wurde von der Schweizerischen
Alzheimervereinigung und vom Alzheimer Forum Schweiz finanziell unterstützt. Frau B. Gilgen unterstützte die Expertengruppe in administrativen Belangen. Ihnen allen herzlichen Dank.

\section{Abstract}

Memory Clinics provide evidence based diagnosis and treatment of dementia. Whenever a diagnosis of dementia is made, it is important to inform the patients about the possible impact of dementia on driving. Patients and their next of kin require competent advice whenever this difficult question is addressed and the mobility desire and the risks related to driving need to be carefully weight up. The time of diagnosis does not necessarily equate to the time when a person with dementia becomes an unsafe driver. The cause and severity of dementia, comorbidities and the current medication need to be carefully taken into account for this decision. On behalf of the association of the Swiss Memory Clinics, a group of experts has developed recommendations to assess fitness to drive in cognitively impaired older adults.

Key words: Memory Clinic - dementia - driving - traffic safety

\section{Résumé}

Les cliniques de la mémoire sont spécialisées dans le diagnostic et la thérapie des démences. Lorsqu'elles posent le diagnostic de démence, elles sont obligées d'informer les conducteurs de véhicules au sujet des possibles consé- quences de la maladie sur la sécurité routière. La conduite automobile soulève des questions délicates, surtout quand il s'agit de pondérer entre le besoin de mobilité du patient et les risques associés à la conduite. De ce fait, les patients et les membres de la famille ont besoin d'une consultation et d'informations précises. Le moment du diagnostic d'une démence n'est pas forcément identique avec celui de l'arrêt de la conduite. La décision quant aux critères médicaux minimaux pour interdire la conduite est difficile à prendre. Il faut considérer le type et la sévérité de la démence, les comorbidités et la médication. Sur mandat de l'association des «Swiss Memory Clinics», un groupe d'experts a développé des recommandations pratiques pour l'évaluation de la capacité de conduire destinées aux cliniques de la mémoire. Basées sur l'expérience clinique et l'évidence publiée, ces recommandations permettent de se faire un jugement plus valable.

Mots-clés: clinique de la mémoire démences - conduite automobile sécurité routière

\section{Korrespondenzadresse}

Prof. Dr. med. Urs P. Mosimann

Direktion Alterspsychiatrie

Universitäre Psychiatrische Dienste UPD

Murtenstrasse 21

3010 Bern

urs.mosimann@gef.be.ch 


\section{Bibliographie}

1. Monsch AU, Hermelink M, Kressig RW, Fisch HP, Grob D, Hiltbrunner B, Martensson B, Rügger-Frey B, von Gunten A: Konsensus zur Diagnostik und Betreeung von Demenzkranken in der Schweiz. Schweiz Med Forum 2008; 8: 144-149.

2. Hort J, O'Brien JT, Gainotti G, Pirttila T, Popescu BO, Rektorova I, Sorbi $S$, Scheltens P: EFNS guidelines for the diagnosis and management of Alzheimer's disease. Eur J Neurol 2010; 10: 1236-1248.

3. Wagner JT, Muri RM, Nef T, Mosimann UP: Cognition and driving in older persons. Swiss Med Wkly 2011; 140: W13136.

4. Kraft E, Marti M, Werner S, Sommer H: Cost of dementia in Switzerland. Swiss Med Wkly 2010; 140: 1-7.

5. Achermann Stürmer Y, Allenbach R, Niemann S, Scaramuzza G, Hayoz R, Dähler-Sturny C: Sinus Report 2011 - Sicherheitsniveau und Unfallgeschehen im Strassenverkehr 2009. Unfallverhütung bBf, editor. Bern, 2011.

6. Seeger R: Fahreignung bei kognitiven Einschränkungen - die möglichst freiwillige Abgabe des Führerausweises ist das Ziel. Hausarzt Praxis 2010; 20: 31-33.

7. Freeman EE, Gange SJ, Munoz B, West SK: Driving status and risk of entry into long-term care in older adults. Am J Public Health 2006; 7: 1254-1259.

8. Ragland DR, Satariano WA, MacLeod KE: Driving cessation and increased depressive symptoms. J Gerontol A Biol Sci Med Sci 2005; 3 : 399-403.

9. Iverson DJ, Gronseth GS, Reger MA, Classen S, Dubinsky RM, Rizzo M: Practice parameter update: evaluation and management of driving risk in dementia: report of the Ouality Standards Subcommittee of the American Academy of Neurology. Neurology 2010; 16: 1316-1324.

10. Martin AJ, Marottoli R, O'Neill D: Driving assessment for maintaining mobility and safety in drivers with dementia. Cochrane Database Syst Rev 2009 (1): CDoo6222.

11. Schwartzberg JG, editor: Physician's guide to assessing and counceling older drivers. Chicago: American Medical Association, 2010.

12. Morris JC, Mohs RC, Rogers H, Fillenbaum G, Heyman A: Consortium to establish a registry for Alzheimer's disease (CERAD) clinical and neuropsychological assessment of Alzheimer's disease. Psychopharmacol Bull 1988; 4: 641-652.

13. Reitan RM: The relation of the trail making test to organic brain damage. J Consult Psychol 1955; 5:393-394.

14. Mathias JL, Lucas LK: Cognitive predictors of unsafe driving in older drivers: a meta-analysis. Int Psychogeriatr 2009; 4: 637-653.

15. Stutts JC, Stewart JR, Martell C: Cognitive test performance and crash risk in an older driver population. Accid Anal Prev 1998; 3: 337-346.

16. Folstein MF, Folstein SE, McHugh PR: «Mini-mental state». A practical method for grading the cognitive state of patients for the clinician. J Psychiatr Res 1975; 3: 189-198.

17. Freund B, Gravenstein S, Ferris R, Burke BL, Shaheen E: Drawing clocks and driving cars. J Gen Intern Med 2005; 3: 240-244.

18. Bukasa B: Das neue Multimedia-Testgerät für die Fahreignungsbegutachtung. In: Meyer-Gramcko F, editor. Verkehrspsychologie auf neuen Wegen: Herausforderungen von Strasse, Wasser, Luft und Schiene. Bonn: Deutscher Psychologen Verlag, 1999: 381-401.

19. Zimmermann P, Sprengelmeyer R, Fimm B, Wallesch CW: Cognitive slowing in decision tasks in early and advanced Parkinson's disease. Brain Cogn 1992; 1: 60-69.
20. Ball K, Owsley C: The useful field of view test: a new technique for evaluating age-related declines in visual function. J Am Optom Assoc 1993 1: 71-79.

21. Clay OJ, Wadley VG, Edwards JD, Roth DL, Roenker DL, Ball KK: Cumulative meta-analysis of the relationship between useful field of view and driving performance in older adults: current and future implications. Optom Vis Sci 2005; 8: 724-731.

22. Wadley VG, Okonkwo O, Crowe M, Vance DE, Elgin JM, Ball KK, Owsley C: Mild cognitive impairment and everyday function: an investigation of driving performance. J Geriatr Psychiatry Neurol 2009; 2:87-94.

23. Frittelli C, Borghetti D, ludice G, Bonanni E, Maestri M, Tognoni G, Pasquali L, ludice A: Effects of Alzheimer's disease and mild cognitive impairment on driving ability: a controlled clinical study by simulated driving test. Int J Geriatr Psychiatry 2009; 3: 232-238.

24. Morris JC: The Clinical Dementia Rating (CDR): current version and scoring rules. Neurology 1993; 11: 2412-2414.

25. Man-Son-Hing M, Marshall SC, Molnar FJ, Wilson KG: Systematic review of driving risk and the efficacy of compensatory strategies in persons with dementia. J Am Geriatr Soc 2007; 6: 878-884.

26. Duchek JM, Carr DB, Hunt L, Roe CM, Xiong C, Shah K, Morris JC: Longitudinal driving performance in early-stage dementia of the Alzheimer type. J Am Geriatr Soc 2003; 10: 1342-1347.

27. Ott BR, Heindel WC, Papandonatos GD, Festa EK, Davis JD, Daiello LA, Morris JC: A longitudinal study of drivers with Alzheimer disease. Neurology 2008; 14: 1171-1178.

28. Brown LB, Ott BR, Papandonatos GD, Sui Y, Ready RE, Morris JC: Prediction of on-road driving performance in patients with early Alzheimer's disease. J Am Geriatr Soc 2005; 1: 94-98.

29. Olde Rikkert MG, Tona KD, Janssen L, Burns A, Lobo A, Robert P, Sartorius $\mathrm{N}$, Stoppe G, Waldemar G: Validity, reliability, and feasibility of clinical staging scales in dementia: a systematic review. Am J Alzheimers Dis Other Demen 2011; 5:357-365.

30. Dawson JD, Anderson SW, Uc EY, Dastrup E, Rizzo M: Predictors of driving safety in early Alzheimer disease. Neurology 2009; 6: 521-527.

31. Rascovsky K, Hodges JR, Knopman D, Mendez MF, Kramer JH, Neuhaus J, et al.: Sensitivity of revised diagnostic criteria for the behavioural variant of frontotemporal dementia. Brain 2011 ; 9: 2456-2477.

32. de Simone V, Kaplan L, Patronas N, Wassermann EM, Grafman J: Driving abilities in frontotemporal dementia patients. Dement Geriatr Cogn Disord 2007; 1: 1-7.

33. Neary D, Snowden JS, Gustafson L, Passant U, Stuss D, Black S, Freedman M, Kertesz A, Robert PH, Albert M, Boone K, Miller BL, Cummings J, Benson DF: Frontotemporal lobar degeneration: a consensus on clinical diagnostic criteria. Neurology 1998; 6: 1546-1554.

34. Rebok GW, Bylsma FW, Keyl PM, Brandt J, Folstein SE: Automobile driving in Huntington's disease. Mov Disord 1995; 6: 778-787.

35. Rizzo M, Uc EY, Dawson J, Anderson S, Rodnitzky R: Driving difficulties in Parkinson's disease. Mov Disord 2010; 25 Suppl 1: S136-140.

36. Cubo E, Martinez Martin P, Gonzalez M, Bergareche A, Campos V, Fernandez JM, Alvarez $M$, Bayes $A$ : What contributes to driving ability in Parkinson's disease. Disabil Rehabil 2010; 5: 374-378.

37. Amick MM, Grace J, Ott BR: Visual and cognitive predictors of driving safety in Parkinson's disease patients. Arch Clin Neuropsychol 2007; 8: $957-967$. 
38. Amick MM, D’Abreu A, Moro-de-Casillas ML, Chou KL, Ott BR: Excessive daytime sleepiness and on-road driving performance in patients with Parkinson's disease. J Neurol Sci 2007; 1: 13-15.

39. Stolwyk RJ, Charlton JL, Triggs TJ, lansek R, Bradshaw JL: Neuropsychological function and driving ability in people with Parkinson's disease. J Clin Exp Neuropsychol 2006; 6: 898-913.

40. Devos H, Vandenberghe W, Nieuwboer A, Tant M, Baten G, De Weerdt W: Predictors of fitness to drive in people with Parkinson disease. Neurology 2007; 14: 1434-1441.

41. Emre M, Aarsland D, Brown R, Burn DJ, Duyckaerts C, Mizuno Y, et al.: Clinical diagnostic criteria for dementia associated with Parkinson's disease. Mov Disord 2007; 22: 1689-1707.

42. McKeith IG, Dickson DW, Lowe J, Emre M, O'Brien JT, Feldman H, et al.: Diagnosis and management of dementia with Lewy bodies: third report of the DLB Consortium. Neurology 2005; 12: 1863-1872.

43. Devos H, Akinwuntan AE, Nieuwboer A, Truijen S, Tant M, De Weerdt $W$ : Screening for fitness to drive after stroke: a systematic review and meta-analysis. Neurology 2011; 8: 747-756.

44. Michon JA: A critical view of driver behavior models: What do we know, what should we do? Evans L, Schwing RC, editors. New York: Plenum Press, 1985.

45. Cotrell V, Wild K: Longitudinal study of self-imposed driving restrictions and deficit awareness in patients with Alzheimer disease. Alzheimer Dis Assoc Disord 1999; 3: 151-156.

46. Wild K, Cotrell V: Identifying driving impairment in Alzheimer disease: a comparison of self and observer reports versus driving evaluation. Alzheimer Dis Assoc Disord 2003; 1: 27-34.

47. Orfei MD, Varsi AE, Blundo C, Celia E, Casini AR, Caltagirone $C$, Spalletta G: Anosognosia in mild cognitive impairment and mild Alzheimer's disease: frequency and neuropsychological correlates. Am J Geriatr Psychiatry 2010; 12: 1133-1140.

48. Leicht H, Berwig M, Gertz HJ: Anosognosia in Alzheimer's disease: the role of impairment levels in assessment of insight across domains. J Int Neuropsychol Soc 2010; 3: 463-473.

49. Herrmann N, Rapoport MJ, Sambrook R, Hebert R, McCracken P, Robillard A: Predictors of driving cessation in mild-to-moderate dementia. CMAJ 2006; 6: 591-595.

50. Cummings JL: The Neuropsychiatric Inventory: assessing psychopathology in dementia patients. Neurology 1997; 5 Suppl 6: S10-16.

51. Verordnung über die Zulassung von Personen und Fahrzeugen zum Strassenverkehr (Verkehrszulassungsverordnung), (1976 [2009]).

52. Gray R, Perkins SA, Suryakumar R, Neuman B, Maxwell WA: Reduced effect of glare disability on driving performance in patients with blue light-filtering intraocular lenses. J Cataract Refract Surg 2011; 1: 38-44.

53. Desapriya E, Wijeratne H, Subzwari S, Babul-Wellar S, Turcotte K, Rajabali F, Kinney J, Pike I: Vision screening of older drivers for preventing road traffic injuries and fatalities. Cochrane Database Syst Rev 2011 (3): CDoo6252.
54. Lehmann R, Fischer-Taeschler D, Iselin HU, Pavan M, Pralong F, Seeger $\mathrm{R}$, Suter S: Richtlinien bezüglich Fahreignung bei Diabetes mellitus. Schweiz Med Forum 2011; 15: 273-275.

55. Seeger R, Lehmann R: Fahreignung und Fahrfähigkeit bei Diabetes mellitus. Therapeutische Umschau 2011; 5: 249-252.

56. Krämer G, Bonetti C, Despland PA, Markoff R, Mathis J, Medici V, Mey er K, Seeger R, Wieser HG: Epilepsie und Autofahren - Richtlinien zur Fahrtauglichkeit der Verkehrskommission der Schweizerischen Liga gegen Epilepsie. Schweizerische Liga gegen Epilepsie (2006).

57. Seeger R: Verkehrsmedizin in der Grundversorger-Praxis. Therapeutische Umschau 2011; 5: 235-241.

58. Mathis J, Seeger R, Kehrer P, Wirtz G: Fahreignung bei Schläfrigkeit: Empfehlungen für Ärzte bei der Betreuung von Patienten mit vermehrter Schläfrigkeit. Schweiz Med Forum 2007; 7: 328-332.

59. Mitler MM, Doghramji K, Shapiro C: The maintenance of wakefulness test: normative data by age. J Psychosom Res 2000; 5: 363-365.

60 . Johns MW: A new method for measuring daytime sleepiness: the Epworth sleepiness scale. Sleep 1991; 6: 540-545.

61. Mathis J: Fahreignung bei Tagesschläfrigkeit. Therapeutische Umschau 2011; 5: 253-255.

62. Brunnauer A, Laux G, David I, Fric M, Hermisson I, Moller HJ:The impact of reboxetine and mirtazapine on driving simulator performance and psychomotor function in depressed patients. J Clin Psychiatry 2008; 12: 1880-1886.

63. Brunnauer A, Laux G, Geiger E, Soyka M, Moller HJ: Antidepressants and driving ability: results from a clinical study. J Clin Psychiatry 2006; 11: 1776-1781.

64. Rapoport MJ, Lanctot KL, Streiner DL, Bedard M, Vingilis E, Murray B Schaffer A, Shulman KI, Herrmann N: Benzodiazepine use and driving: a meta-analysis. J Clin Psychiatry 2009; 5: 663-673.

65. Verster JC, Mets MA: Psychoactive medication and traffic safety. Int J Environ Res Public Health 2009; 3: 1041-1054.

66. Brunnauer A, Laux G, Zwick S: Driving simulator performance and psychomotor functions of schizophrenic patients treated with antipsychotics. Eur Arch Psychiatry Clin Neurosci 2009; 8: 483-489.

67. Iwersen-Bergmann S, Andresen $\mathrm{H}$, Puschel K, Heinemann A, von Renteln-Kruse W: Use of psychotropic substances by the elderly and driving accidents. Z Gerontol Geriatr 2009; 3: 193-204.

68. Strassenverkehrsgesetz (SVG), (1958)

69. Canadian Medical Association C: Determining medical fitness to operate motor vehicles. Association CM, editor. Ottawa: Canadian Medical Association, 2006 